\title{
A Novel LncRNA-miRNA-mRNA Triple Network Identifies LncRNA RP11-363E7.4 as An Important Regulator of miRNA and Gene Expression in Gastric Cancer
}

\author{
Pengcheng Wang ${ }^{\mathrm{a}} \quad$ Jing $\mathrm{Li}^{\mathrm{b}} \quad$ Wei Zhao $^{\mathrm{c}} \quad$ Chunyang Shang $^{\mathrm{a}} \quad$ Xian Jiang $^{\mathrm{a}}$ \\ Yuli Wanga Baoguo Zhou ${ }^{a}$ Fusheng Bao ${ }^{d}$ Haiquan Qiao \\ aDepartment of General Surgery, The First Affiliated Hospital of Harbin Medical University, Harbin, \\ ${ }^{b}$ College of Bioinformatics Science and Technology, Harbin Medical University, Harbin, 'Department \\ of Anesthesiology, The First Affiliated Hospital of Harbin Medical University, Harbin, dDepartment of \\ General Surgery, Mingshui Country People's Hospital, Suihua, P.R. China
}

\section{Key Words}

Gastric cancer - LncRNAs-miRNA-mRNA regulators - Triple network analyses - Function enrichment $\cdot$ Random walk

\begin{abstract}
Background/Aims: Recent evidence has shown that some long non-coding RNAs (IncRNAs) play important roles in various biological processes. However, the regulatory mechanism of IncRNA in gastric cancer (GC) remains unclear. Methods: We reannotated the GC gene expression profile into a IncRNA-mRNA biphasic profile and integrated the microRNA target data to construct a global GC triple network. A further clustering and random walk with restart analyses was performed on the triple network from the level of topology analyses. Quantitative real-time PCR was used to determine expression of IncRNA RP11-363E7.4. Kaplan-Meier analyses was performed to evaluate the prognostic value of IncRNA RP11363E7.4. Results: We constructed a gastric cancer IncRNA-miRNA-mRNA network (GCLMN) including six IncRNAs, 332 mRNAs, and 3,707 edges. For the shared IncRNA RP11-363E7.4, the interacting gene and microRNA functional enrichment studies implied that IncRNA RP11363E7.4 might function as a new regulator in GC. The expression of IncRNA RP11-363E7.4 was downregulated compared with that of paracarcinoma tissues in five GC samples. High expression of IncRNA RP11-363E7.4 was found to be correlated to better overall survival (OS) for GC patients. Conclusions: This study focused on GC IncRNA-miRNA-mRNA regulatory networks, and found that IncRNA RP11-363E7.4 was a new GC risk IncRNA, which might provide novel insight into a better understanding of the pathogenesis of GC.
\end{abstract}




\section{Cellular Physiology Cell Physiol Biochem 2018;47:1025-1041 \\ \begin{tabular}{l|l} 
and Biochemistry Published online: May 30, 2018 & $\begin{array}{l}\text { C } 2018 \text { The Author(s). Published by S. Karger AG, Basel } \\
\text { www.karger.com/cpb }\end{array}$
\end{tabular} \\ Wang et al.: Network Analyses Identifies IncRNA RP11-363E7.4 as an Important Gastric Cancer Regulator}

\section{Introduction}

Gastric cancer (GC) is the fifth most common cancer and the most frequent cause of cancer-related deaths worldwide [1,2]. It is a molecular disease related to the imbalance of oncogenes and suppressor genes. Although studies have provided novel insights into the mechanisms of GC carcinogenesis [3-5], the exact cause remains unclear. Thus, there is an urgent need to elucidate the etiological mechanisms and develop novel therapeutics to treat GC.

Non-coding RNAs (ncRNAs) were reported to be transcriptionally nonfunctional because they have no protein coding function. With improved technologies, studies have suggested that the ncRNAs act as key regulators and may play important biological roles in various cellular processes, such as gene expression, cell proliferation, differentiation, and apoptosis [6-9]. Long non-coding RNAs (lncRNAs) are members of the ncRNA group, having transcripts of approximately 200 nucleotides or less. LncRNAs are critical in gene regulatory networks, and act at the transcriptional, posttranscriptional, and epigenetic levels through cis- or trans-acting pathways [10-12].

Recently, studies have suggested that lncRNAs play important roles in many complicated cancers, including cervical cancer [13], lung cancer [14], breast cancer [15], esophageal cancer [16], hepatocellular carcinoma [17], colorectal cancer [18, 19], and GC [20-22]. The discovery of competing endogenous RNAs (ceRNAs) led to the hypothesis that other RNAs could interact with each other through microRNA (miRNA) response elements [23]. Crosstalk at the transcriptional level exists between mRNAs and ncRNAs, such as lncRNAs, circular RNAs, and pseudogenes. Zhao et al. reported that the expression of small nucleolar RNA host gene (5SNHG5) was significantly downregulated in GC tissues and SNHG5 could play a suppressor role by targeting metastasis-associated protein 2 (MTA-2) [24]. Zhao et al. also reported another function of SNHG5 in GC, suggesting that it acted as a binding site for miR-32 to modulate the suppression of Kruppel-like factor 4 (KLF-4) [25]. Another study demonstrated that the IncRNA, phosphatase and tensin homolog pseudogene 1 (PTENP1), was downregulated in GC tissues, and overexpression of PTENP1 inhibited cell growth, induced apoptosis, and suppressed migration and invasion in GC cell lines. The specific mechanism of PTENP1 may include its function as a ceRNA to modulate PTEN1 levels through miR-106b and miR-93 [26]. Despite these previous studies, our knowledge about the roles of IncRNA-miRNA-mRNA networks in the pathogenesis of GC is still limited.

In this study, we constructed a GC-related IncRNA-mRNA network (GCLMN) by mapping the differentially expressed lncRNAs and mRNAs into an established global triple network. We determined that IncRNA RP11-363E7.4 was a key regulator both in the topology and random walk with a restart (RWR) analyses. Then, we performed quantitative real-time PCR to validate the expression of lncRNA RP11-363E7.4 by extracting five additional clinical GC tissue samples, and evaluated its prognostic value by Kaplan-Meir analyses for GC. This novel approach of predicting GC related IncRNA and IncRNA-miRNA-mRNA networks could help to understand the underlying mechanism of GC.

\section{Materials and Methods}

Gene expression profile probe reannotation

The gene expression profile GSE19826 in the present study was obtained from the GEO database, which contained 12 paired adjacent normal and tumor-matched gastric tissues and three nonadjacent normal mucosal tissues [27]. All tissue samples were obtained from Chinese patients with stage I-IV GC. The gene expression platform was GPL570.

The transcript sequences of the IncRNAs and proteins were downloaded from the GENCODE database (http://www.gencodegenes.org/). The probe annotation sequences were obtained from Affymetrix (http:// www.affymetrix.com/). By using the Blastn tools, we identified the probe-matched lncRNA and protein coding sequences. By introducing the following criteria, we obtained the ideal alignment results: (1) reserve 


\section{Cellular Physiology Cell Physiol Biochem 2018;47:1025-1041 \begin{tabular}{ll|l} 
DOI: 10.1159/000490168 & O 2018 The Author(s). Published by S. Karger AG, Basel \\
www.karger.com/cpb
\end{tabular} \\ Wang et al.: Network Analyses Identifies IncRNA RP11-363E7.4 as an Important Gastric Cancer Regulator}

the probes that uniquely matched to protein coding transcripts or IncRNA transcripts; (2) reserve probes that matched to only one transcript in probe-transcript pairs; (3) reserve transcripts that perfectly matched to more than three probes [28].

\section{Identification of the differential expression gene with SAM}

The significance analyses of microarrays (SAM) is one of the most frequently utilized methods for gene expression data analyses [29]. The SAM identifies genes with statistically significant changes in expression by combining the gene-specific $t$-test with a statistical $d$ value. In this study, we applied SAM to discover differentially expressed genes in 27 samples. A $d$ value of $<0.01$ was considered statistically significant.

\section{The mRNA and IncRNA targeted miRNA extractions}

Starbase V2.0 (http://starbase.sysu.edu.cn/) is a powerful dataset tool used to clarify the regulatory interaction networks among miRNAs, IncRNAs, pseudogenes, circular (circ) RNAs, and ceRNAs from 108 CLIP-Seq (PAR-CLIP, HITS-CLIP, iCLIP, and CLASH) data sets [30]. In this study, the miRNA-mRNA interactions and miRNA-IncRNA interactions were obtained from this database. We identified 423, 975 miRNA-mRNA interaction pairs, including 13, 805 mRNAs, 386 miRNAs, and 10, 212 miRNA-IncRNA interaction pairs (including 1, 127 lncRNAs and 277 miRNAs).

Hypergeometric test to identify IncRNA-mRNA interactions

In this study, the GCLMN was constructed by using the hypergeometric test as follows:

$$
p=1-\sum_{k=0}^{r-1} \frac{\left(\begin{array}{l}
t \\
k
\end{array}\right)\left(\begin{array}{l}
T-t \\
N-k
\end{array}\right)}{\left(\begin{array}{l}
T \\
N
\end{array}\right)}
$$

where $\mathrm{T}$ was the total counts of human miRNA; $t$ designated the number of miRNA interacting with the mRNA; $\mathrm{N}$ denoted the number of miRNA interacting with the lncRNA; and $r$ represented the number of miRNA shared by the mRNA and IncRNA. A $p$ value of $<0.01$ was considered statistically significant.

\section{RWR to prioritize GC related IncRNAs}

To prioritize GC-related IncRNAs, we performed RWR algorithm analyses with GCLMN [31, 32]. This method simulates a walker that randomly moves from the current node to neighboring nodes with a probability ( $\mathrm{r}$ ) in the walk [32]. It was described as follows:

$\mathrm{P}^{\mathrm{t}+1}=(1-\mathrm{r}) \mathrm{WP}^{\mathrm{t}}+\mathrm{rP}^{0}$

where $\mathrm{P}^{\mathrm{t}}$ was a vector in which the ith element holds the probability of discovering the random walker at node $i$ at step t. $\mathrm{P}^{0}$ was the initial probability vector. $\mathrm{W}$ represented the column normalized adjacency matrix of the network, and $r$ was the restart probability of the random walk at every step at the source nodes. When the difference between $\mathrm{P}^{\mathrm{t}}$ and $\mathrm{P}^{\mathrm{t}+1}$ fell below a given cutoff value, the update procedure was finished, and $\mathrm{P}^{\mathrm{t}+1}$ was the output value of the RWR algorithm.

In this study, by retrieving relevant previous reports through the keywords "gastric cancer and RNA," we finally selected four cancer-related protein coding genes, which have been tested by different kinds of experiments. These four genes ("NOV," "KIT," "SP1," and "CLOCK") were considered as seeds in the RWR model [33-36]. The initial probability $\mathrm{P}^{0}$ of each seed mRNA was set as $1 / \mathrm{n}$ (where $\mathrm{n}$ is the number of seed mRNAs), while other non-seed mRNAs were set as 0 . The $r$ was set to 0.5 . The stable probability Po of each non-seed mRNA could be obtained when the difference between $\mathrm{P}^{t}$ and $\mathrm{P}^{\mathrm{t}+1}$ was less than $10^{-10}$. All candidate lncRNAs could be sorted based on Po, and IncRNAs with high scores were considered as the most potentially disease-related IncRNAs. The statistical significance was determined by comparing the scores of the IncRNAs in the network following 3, 000 iterations of the known GC-related gene shufflings, while keeping the network topological properties consistent [28]. The rank proportion of the score gained from the actual network for each IncRNA in the 3, 000 iterations is listed as a statistical significant $p$ value.

\section{KARGER}




\section{Cellular Physiology Cell Physiol Biochem 2018;47:1025-1041 \\ \begin{tabular}{l|l|l} 
DOI: 10.1159/000490168 & $\begin{array}{l}\text { O 2018 The Author(s). Published by S. Karger AG, Basel } \\
\text { wwww.karger.com/cpb }\end{array}$
\end{tabular} \\ Wang et al.: Network Analyses Identifies IncRNA RP11-363E7.4 as an Important Gastric Cancer Regulator}

\section{Topological features of the GCLMN}

The topological structure of the network was further analyzed based on the degree of the node, betweenness, and closeness. For a given graph, $G=(V, E), V$ and $E$, respectively, represented a set of nodes and edges. The degree (D) is generally used to reflect the basic topological property in the network and represents the number that edges directly link to the node V. In our study, K was the number of edges linked to node V. It was described as follows:

$\mathrm{D}(\mathrm{v})=\mathrm{K}$

Betweenness centrality (BC) is one of the most effective approaches to evaluate the importance of one node in the network. The higher the betweenness, the more important the node exerted close connectivity in the network. It was defined as:

$$
B C(v)=\sum s \neq v \neq f \frac{\sigma_{s f}(v)}{\sigma_{s f}}
$$

where $\delta_{s f}$ represented the shortest path from node s to node f, and $\delta_{s f}(v)$ was the node count $(v)$ from node $s$ to node $\mathrm{f}$.

Closeness ( $\mathrm{C}$ ) represented the average distance from one node to all other nodes in the network, and it reflected the extent from one node to the "network centrality." The specific calculating formula is as follows:

$$
C(v)=\frac{1}{\sum_{k=1}^{n} d(j, v)}
$$

where $d(j, v)$ denoted the shortest distance from $j$ to $v$, and $n$ represented the number of nodes of the network.

\section{Functional enrichment analyses}

To realize the enrichment analyses of candidate feature genes, GO and KEGG analyses were performed using DAVID, which provide a comprehensive set of functional annotation tools for investigators to understand biological meaning behind large list of genes [37]. In this study, $p<0.05$ was considered statistically significant.

\section{Patients and specimens}

The GC samples and corresponding non-cancerous specimens were obtained from five patients who underwent surgery at the First Affiliated Hospital of Harbin Medical University, from February to April in 2017. These patients were separate from the GSE set and were used as a validation cohort. All participants received no other therapies before surgery and provided written informed consent before sample collection. The present study protocol was approved by the Human Research Ethics Committee of the hospital. All GC samples were diagnosed by two individual pathologists based on the World Health Organization classification system. All samples were preserved in liquid nitrogen and then stored at $-80^{\circ} \mathrm{C}$ until use.

\section{RNA isolation and quantitative RT-PCR}

Total RNA was extracted from 10 samples (five GC tissues and five matched-adjacent tissues) using TRIzol'"' reagent (Tiangen, Beijing, China) according to the manufacturer's instructions. The RNA quantity and quality were confirmed with a NanoDrop ND-2000 spectrophotometer (ThermoFisher, Scotts Valley, CA, USA). RNA integrity was assessed by using standard denatured agarose gel electrophoresis.

Reverse transcription reactions were performed using the PrimeScript"' RT Reagent kit (Takara, Mountain View, CA, USA). The quantitative real time PCR analyses was carried out on the Applied Biosystems (Carlsbad, CA, USA) 7500 Real-Time PCR System by using the SYBR ${ }^{\circledR}$ Green Premix Ex Taq II (TaKaRa, Tokyo, Japan), and 18srRNA as the internal control. The relative expression level was determined with the $2^{-\Delta \Delta c t}$ method [38]. Each sample was performed in triplicate. The primer sequences for qRT-PCR in our study were as follows: RP11-363E7.4 forward, 5'-CGACCACCTATTCCACTT-3';

RP11-363E7.4 reverse, 5'-GCCAGGAAGGCTCAAATC-3';

18srRNA forward, $5^{\prime}$ - GTAACCCGTTGAACCCCATT- $3^{\prime}$; and

18srRNA reverse, $5^{\prime}$ - CCATCCAATCGGTAGTAGCG - $3^{\prime}$. 


\section{Kaplan-Meier analyses}

The Kaplan-Meier plotter analyses is a useful online database containing useful clinical information to predict the OS of four common cancers [39]. The prognostic value of LncRNA RP11-363E7.4 (227306_ at) was performed in GC patients, breast cancer patitents and lung cancer patients by using Kaplan-Meier Plotter (http://kmplot.com/analyses/) [39]. The hazard ratio (HR) and log rank $p$ value were determined and displayed on the webpage.

\section{Statistical analyses}

All data are expressed as mean \pm SD and analyzed using GraphPad Prism 5.0 (San Diego California USA). The two-tailed Student's $t$-test was used to compare two different groups. A value of $p<0.05$ was considered statistically significant.

\section{Results}

Identification of GC associated mRNAs and IncRNAs

In the present study, we used a method to simultaneously reannotate lncRNA and mRNA expression. First, we downloaded GSE19826 from the Gene Expression Omnibus (GEO) database, which included 12 matched samples of GC and three nonadjacent normal mucosal tissues. Then, we used BLASTn tools for sequence alignments between annotated probe sequences and human long noncoding transcript sequences and protein coding transcript sequences. Using the filtration set, we eventually identified 139, 180 mRNA probes and 38, 009 IncRNA probes.

To identify differentially expressed lncRNAs and mRNAs, we calculated the 12 matched samples and three normal samples with significance analyses of microarrays (SAM) tests (see the Methods section) after gene expression profile reannotation. In this step, we considered a value of $p<0.05$ to be statistically significant. Finally, there were 1, 577 differentially expressed mRNAs and 141 differentially expressed lncRNAs, which were defined as key GC related mRNAs and lncRNAs.

Construction of the $\mathrm{In}$ cRNA-mRNA regulatory network

In this study, our first goal was to establish a ln-

Table 1. The detailed information top 10 genes in topological analyses. Note: There were 2 IncRNAs (RP11-363E7.4, RP11-834C11.4) and 3 mRNAs (SLC16A10, SMCR8 and ZNF626) appeared in each dimension. The node with significant topological features played important roles in the network

\begin{tabular}{llllll}
\hline Gene & degree & Gene & betweenness & Gene & closeness \\
\hline RP11-363E7.4 & 153 & RP11-363E7.4 & 43500.5 & RP11-363E7.4 & 0.000154775 \\
RP11-834C11.4 & 104 & RP11-834C11.4 & 27387.5 & SLC16A10 & 0.000152114 \\
RP6-24A23.7 & 32 & CTB-31020.2 & 17616 & SMCR8 & 0.000152114 \\
CTB-31020.2 & 32 & ZNF626 & 16384 & ZNF626 & 0.000150331 \\
ZNF503-AS2 & 13 & SLC16A10 & 11124 & RP11-834C11.4 & 0.000149544 \\
SLC16A10 & 2 & SMCR8 & 11124 & TMEM192 & 0.000147493 \\
SMCR8 & 2 & RP6-24A23.7 & 9424 & HIP1R & 0.000147493 \\
ZNF626 & 2 & MLLT11 & 9216 & MCPH1 & 0.000147493 \\
MLLT11 & 2 & ZNF503-AS2 & 78 & WDFY3 & 0.000147493 \\
RP11-613D13.8 & 2 & RP11-613D13.8 & 1 & GABPB2 & 0.000147493 \\
\hline
\end{tabular}

\section{KARGER}


cRNA-mRNA association network. We collected 423, 975 miRNA-mRNA interactions using starBase V2.0. Because miRNAs could interact with lncRNAs, we searched starBase V2.0 for lncRNA-miRNA interactions and finally identified 10, 212 miRNA-lncRNA interaction pairs. Then, these two sets of data were merged to build a IncRNA-miRNA-mRNA network. The nodes in the network were miRNAs, mRNAs, and lncRNAs. The edges in the network represented interactions between these RNAs (Fig. 1).

Next, we mapped 1, 577 GC-associated differentially expressed mRNAs and 141 GCassociated differentially expressed lncRNAs into the network to identify the GC-related IncRNAs. We extracted these key lncRNAs and their associated miRNAs in the network, and performed hypergeometric statistical tests to evaluate the number of shared miRNAs between the lncRNA and mRNA pairs. Finally, we constructed a GCLMN, which consisted of six IncRNAs, 332 mRNAs, and 3, 707 edges (Fig. 2A, Fig. 3-6).

\section{Topological analyses of the GC related IncRNA-mRNA network}

The topological properties of the molecule network included degree, betweenness, and closeness. Therefore, we analyzed these common topological measurements to reveal the characteristics of the GCLMN. Based on the degree, we produced two charts. The degree distribution of mRNA and lncRNA are shown in Fig. 2B and 2C. Moreover, we list the top ten significant genes with high topological features in Table 1. Interestingly, two lncRNAs

Fig. 2. Topological features of the GCLMN. (A) Overview of the GCLMN; the red and orange nodes represented GC-related IncRNAs and mRNAs, respectively. The size of nodes denoted the degrees of the nodes in the network. (B) The node distribution of all mRNAs. (C) The node distribution of all lncRNAs. (D) The Venn diagram analyses showing the overlap of the top 10 max nodes with topological features in each dimension. (E) The significant changes in the GO biological process for IncRNA RP11-363E7.4. (F) The significant changes in the GO biological process for IncRNA RP11-834C11.4. The $\mathrm{x}$-axis was the $-\log 10$ of the $\mathrm{p}$ value, and $\mathrm{p}<0.05$ was considered statistically significant. GCLMN: gastric cancer-related IncRNAmRNA network.

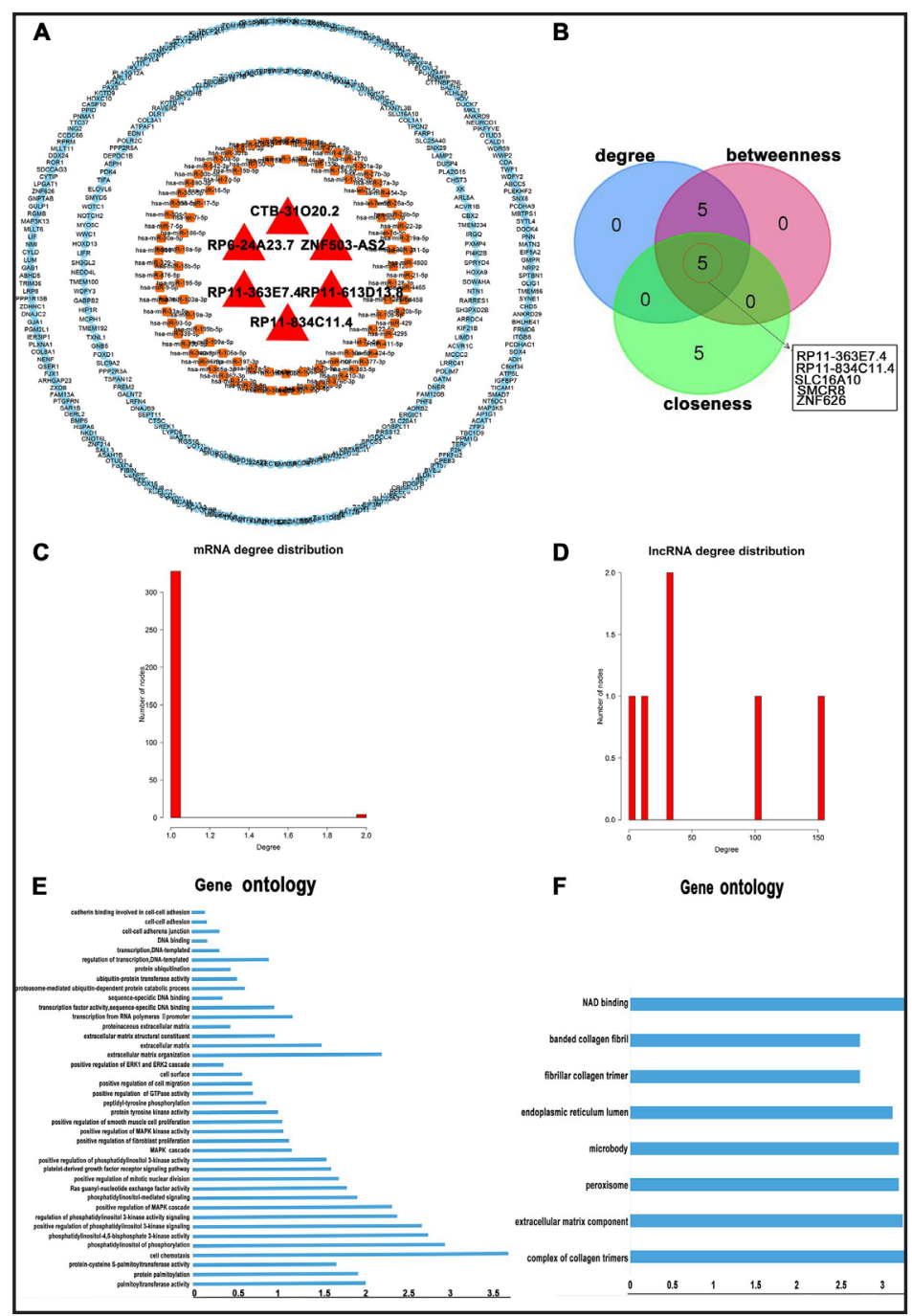




\section{Cellular Physiology and Biochemistry}

Cell Physiol Biochem 2018;47:1025-1041

\begin{tabular}{l|l}
\hline DOI: $10.1159 / 000490168$ & (c) 2018 The Author(s). Published by S. Karger AG, Basel
\end{tabular}

ished online: May 30, 2018 www.karger.com/cpb

1031

Wang et al.: Network Analyses Identifies IncRNA RP11-363E7.4 as an Important Gastric Cancer Regulator

Fig. 3. Cluster analysis of lncRNA CTB-31020.2 and their neighbor mRNAs in GCLMN. (A) The heatmap of lncRNACTB-31020.2 and their neighbor mRNAs based on their expression. The columns represented 27 samples and the rows represented IncRNA and its neighbor mRNAs. (B) The network of IncRNACTB-31020.2 extracted from global triple network. GCLMN: gastric cancer related IncRNA-mRNA network.

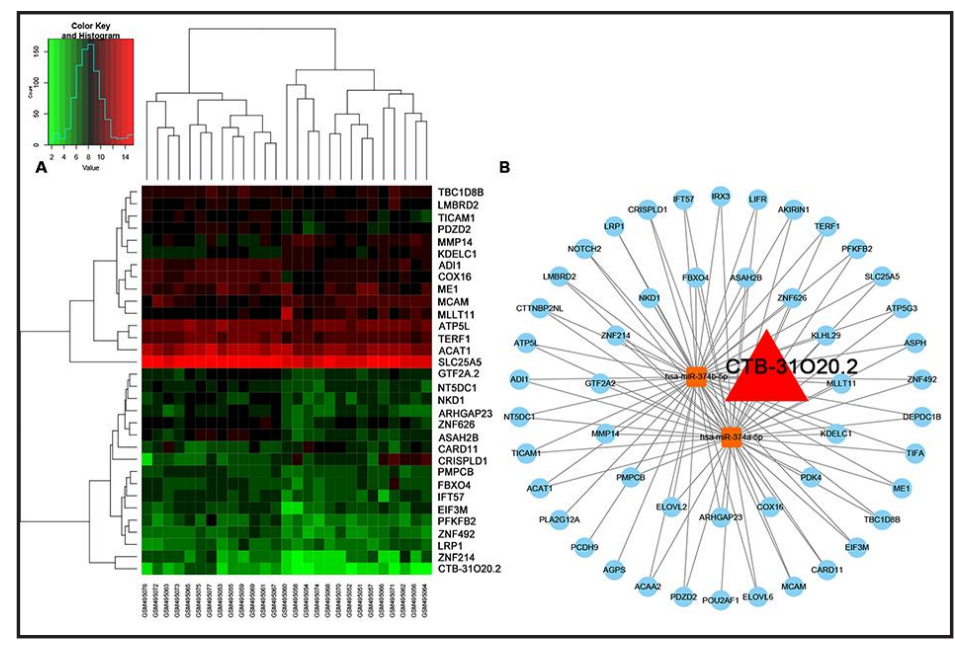

Fig. 4. Cluster analysis of lncRNA ZNF503-AS2 and their neighbor mRNAs in GCLMN. (A) The heatmap of IncRNA ZNF503-AS2 and their neighbor mRNAs based on their expression. The columns represented 27 samples and the rows represented lncRNA and its neighbor mRNAs. (B) The network of lncRNA ZNF503-AS2 extracted from global triple network. GCLMN: gastric cancer related IncRNA-mRNA network.

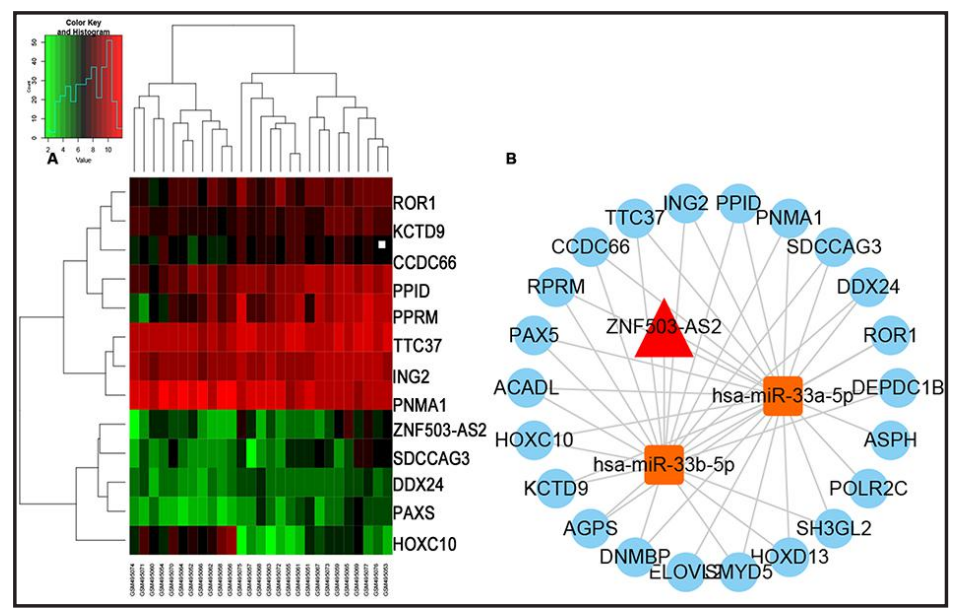

Fig. 5. Cluster analysis of lncRNA RP11-613D13.8 and their neighbor mRNAs in GCLMN. (A) The heatmap of IncRNA RP11-613D13.8 and their neighbor mRNAs based on their expression. The columns represented 27 samples and the rows represented IncRNA and its neighbor mRNAs. (B) The network of IncRNA RP11-613D13.8 extracted from global triple network. GCLMN: gastric cancer related lncRNA-mRNA network.

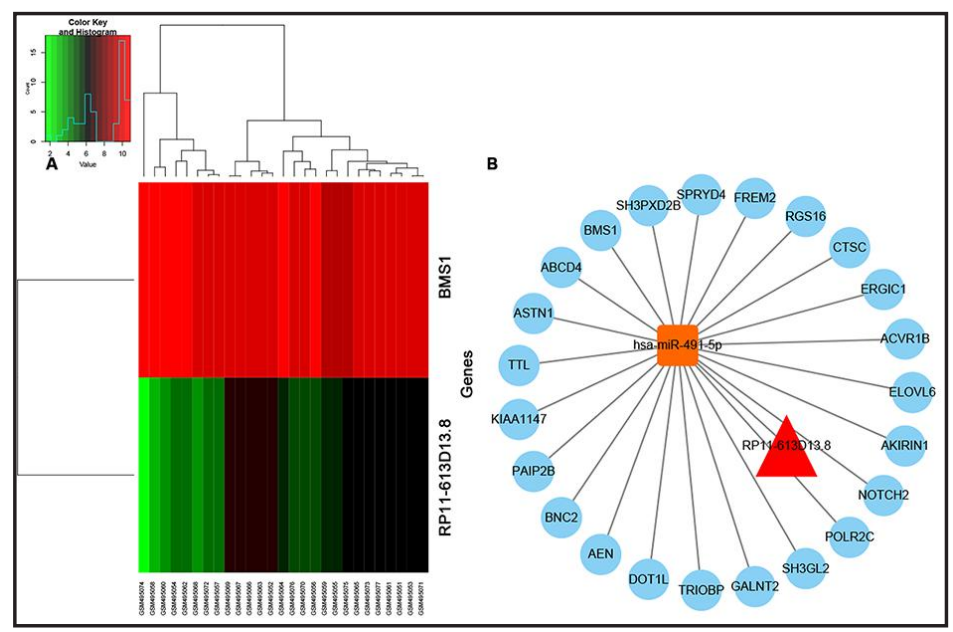

(RP11-363E7.4 and RP11-834C11.4) and three mRNAs (SLC16A10, SMCR8, and ZNF626) were selected in each dimension (Fig. 2D).

To investigate the functions of these two lncRNAs in GC, gene ontology (GO) and the Kyoto Encyclopedia of Genes and Genomes (KEGG) analyses were performed by using their first mRNA neighbors in the GCLMN. The results of GO and KEGG pathway analyses revealed $16 \mathrm{GO}$ terms and two pathways enriched in lncRNA RP11-363E7.4. The results of the GO biological 
Fig. 6. Cluster analysis of lncRNA RP6-24A23.7 and their neighbor mRNAs in GCLMN. (A) The heatmap of IncRNA RP6-24A23.7 and their neighbor mRNAs based on their expression. The columns represented 27 samples and the rows represented IncRNA and its neighbor mRNAs. (B) The detailed module heatmap and the names of IncRNAs and mRNAs were listed on the right table. (B) The network of IncRNA RP6-24A23.7extracted from global triple network. GCLMN: gastric cancer related lncRNA-mRNA network.
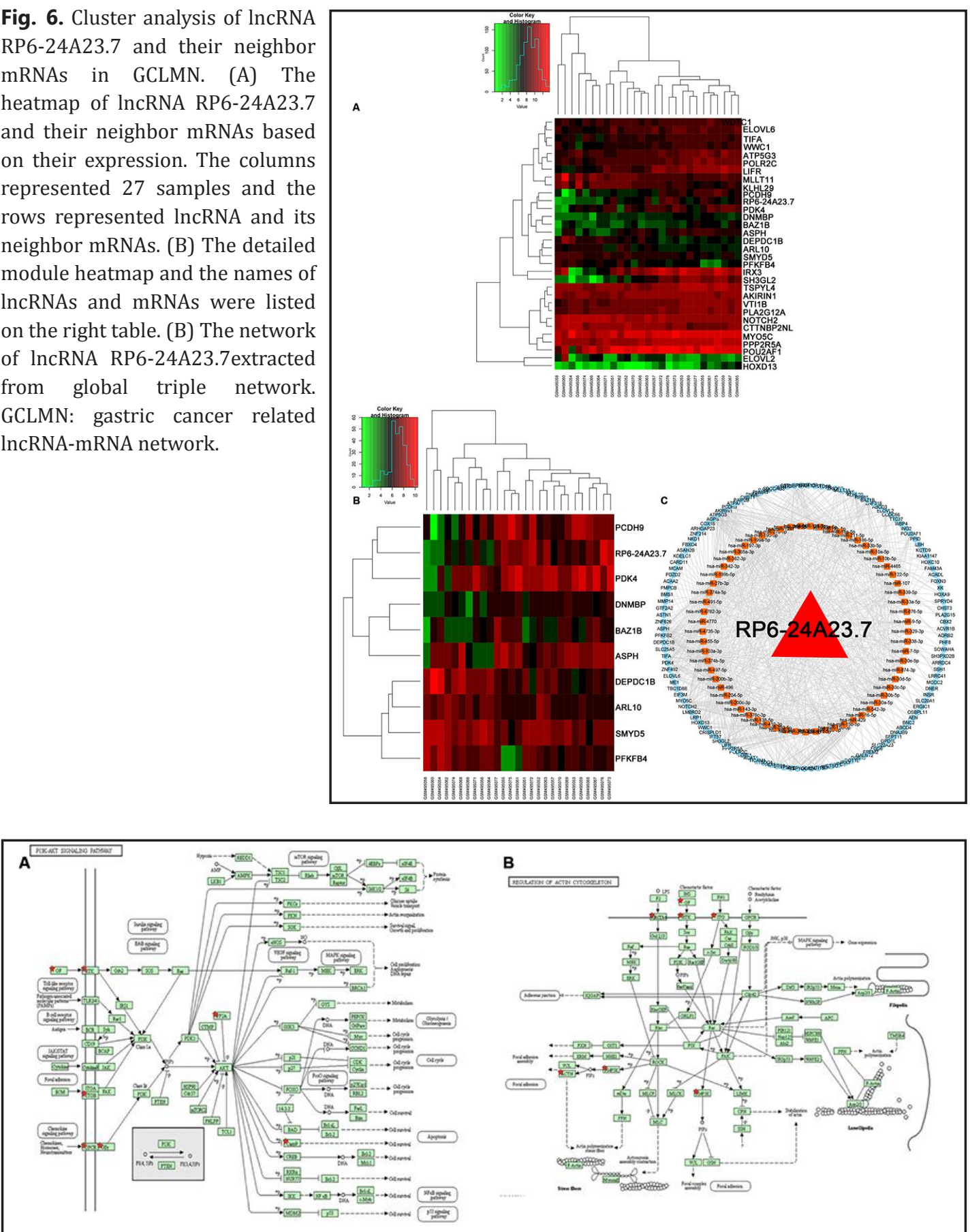

Fig. 7. The KEGG pathway related to IncRNA RP11-363E7.4. (A) PI3K-AKT signaling pathway. (B) Regulation of actin cytoskeleton. Red marks represent the genes that were co-expressed with lncRNAs.

process term analyses showed that the differentially expressed genes were involved in many cellular aspects, such as cell chemotaxis, positive regulation of PI3K signaling, positive regulation of the MAPK cascade, extracellular matrix organization, positive regulation of mitotic nuclear division, and the platelet-derived growth factor receptor signaling pathway (Fig. 2E). The results of KEGG enrichment analyses showed two important cancer-related pathways, the PI3K-Akt signaling pathway and regulation of the actin cytoskeleton (Fig. 7).

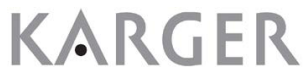


These biological processes and pathways have been reported as highly associated with GC pathogenesis. MAPK signaling pathways play an essential role in regulating cell proliferation and metastasis in GC. Cui et al. found that the expressions of proteins in the MAPK pathway, such as phosphoMEK1/2/MEK1/2, phospho-ERK1/2/ERK1/2, and phospho-c-Jun/c-Jun, were significantly decreased when asparagine endopeptidase (AEP) was decreased, which resulted in decreases in tumor invasion and metastasis [40]. Moreover, Lan et al. have demonstrated that cross-linked hyaluronic acid gels used to prevent postoperative intraperitoneal adhesion could have anti-tumor effects on GC by inhibiting the epidermal growth factor (EGF) -induced signaling of MAPK/ERK, PI3K/Akt, and Rac1 mediated pathways [41]. Similar to the MAPK signal pathway, Rho GTPases play pivotal roles in tumor progression by regulating tumor cell migration and invasion. Fujimoto et al. reported that PAR1 induced GC cells to become stem-like, inhibited yes-associated protein (YAP) phosphorylation, and increased YAP activity via Rho GTPase [42]. Pang et al. reported that thrombin-induced RhoA and Rac1 activation could be significantly inhibited by ALEX1 overexpression, which indicated that ALEX1 could inhibit tumor metastasis through the PAR-1/Rho GTPase signaling pathway [43]. The PI3K pathway plays pivotal roles in many essential cellular processes including cell growth, differentiation, invasion, metastasis, and resistance to chemotherapy [5]. Huang et al. suggested that gelsolin-PI3KAkt signaling was important for GC cell aggregation and may be a promising biomarker for the treatment of GC. Zhu et al. found that S-allylmercaptocysteine suppressed GC cell proliferation, induced cell apoptosis, inhibited the PI3K/Akt pathway, and suppressed tumor growth in SGC-7901 xenografts [44].

Regarding another IncRNA, RP11-834C11.4, functional enrichment analyses showed that the genes in the IncRNA RP11-834C11.4-miRNA-mRNA network were also involved in the carcinogenesis of GC (Fig. 2F).

Our findings suggested that lncRNA RP11-363E7.4 and lncRNA RP11-834C11.4, which had significant topological features, might exert important roles in the carcinogenesis of GC.

Based on the result of topological analyses, we further applied cluster analyses to investigate the functions of these two lncRNAs in the GCLMN. The cluster analyses for IncRNA RP11-834C11.4 is showed in Fig. 8A. The clustering between the lncRNAs and miRNAs is showed in Fig. 8B. We found that let-7, miR-590, miR-27, and the miR-133 family were involved in the subnetwork (Fig. 8C). Studies have demonstrated that these miRNAs were highly related to GC [45-48]. Regarding IncRNA RP11-363E7.4, the heatmap and the module network are shown in Fig. 8D and 8E, respectively. In Fig. 8F, we show that the miRNAs of the subnetwork were highly associated with GC. These results suggested that lncRNA RP11834C11.4 and IncRNA RP11-363E7.4 played an important role in the the development of GC.

\section{Random walk with restart analyses of a GC-related IncRNA-mRNA network}

In our study, we performed a RWR algorithm analyses on a heterogeneous network using four validated cancer-related genes ("NOV," "KIT," "SP1," and "CLOCK") as seed nodes, prioritizing GC related genes. Because not all these genes are tightly associated with GC, a permutation test was also performed. After the 3, 000 times random walk with permutation, we identified two lncRNAs with $p<0.05$. They were lncRNA RP11-613D13.8 and IncRNA RP11-363E7.4, and both were considered as potential regulators of GC. To further investigate the roles of the aforementioned lncRNAs, we constructed two sub-networks based on the lncRNA-miRNA-mRNA model (Fig. 9A, 9B), and the role of these two lncRNAs was assessed, while examining neighboring miRNAs. As a result, we showed that lncRNA RP11-613D13.8 interacted with miR-491-5p. Sun et al. reported that the expression of miR-491-5p was downregulated in GC tissues. In addition, forced expression of miR-491-5p inhibited tumor proliferation, disrupted the cell cycle, and induced apoptosis in vitro and in vivo [49]. We also showed that lncRNA RP11-363E7.4 interacted with three miRNAs (miR-17-5p, miR-19b-3p, and miR-106a-5p), which were previously investigated in the reports of Xia et al., Zhang et al., and Qu et al. [22, 50, 51].

\section{KARGER}




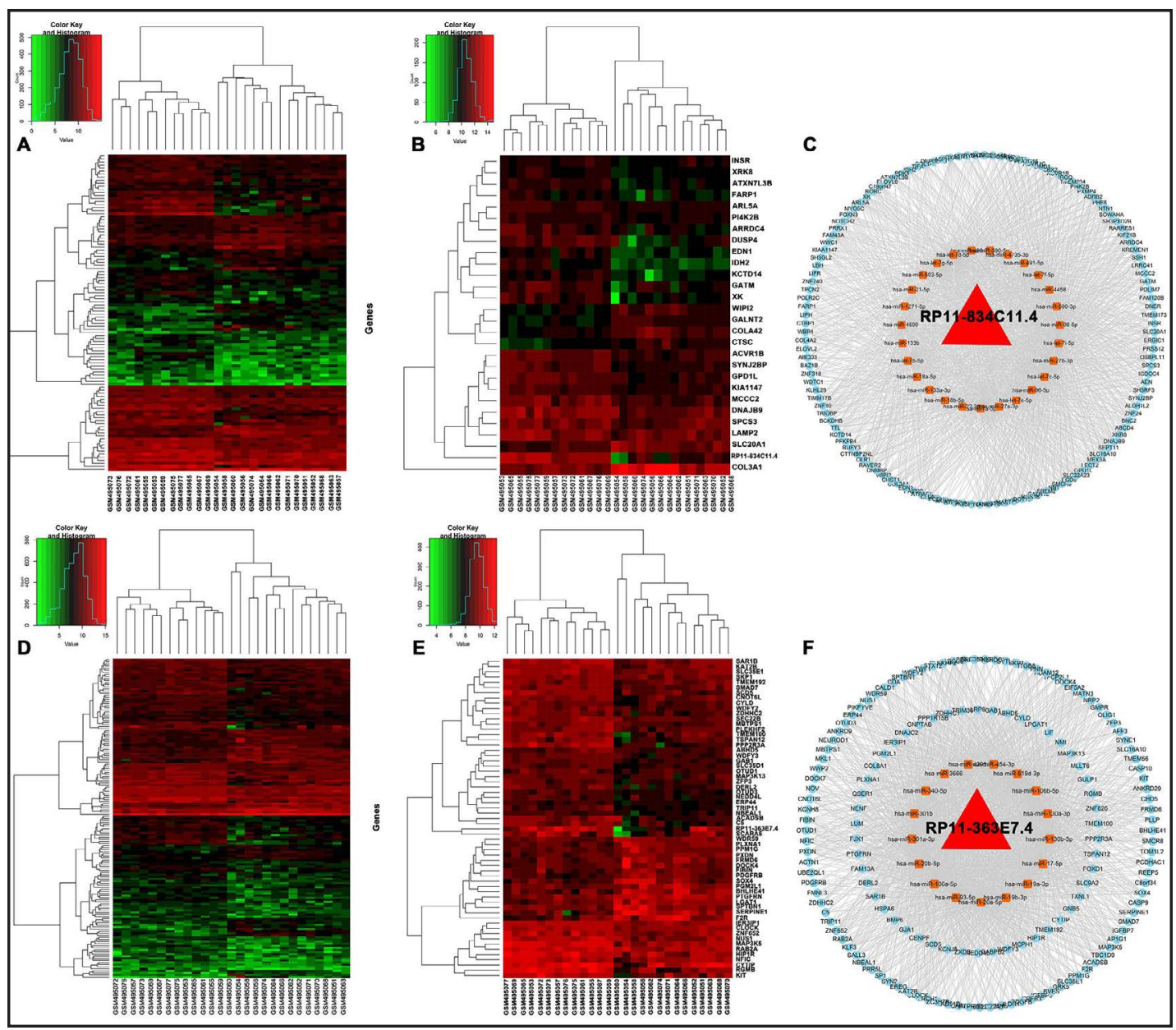

Fig. 8. The regulatory module of IncRNA RP11-834C11.4 and IncRNA RP11-363E7.4 in GCLMN. (A) The heatmap of IncRNA RP11-834C11.4 and their neighbor mRNAs based on their expression. (B) The detailed module heatmap of IncRNA RP11-834C11.4. (C) The IncRNA RP11-834C11.4-miRNA-mRNA network extracted from global triple network. (D) The heatmap of lncRNA RP11-363E7.4 and their neighbor mRNAs based on their expression. (E) The detailed module heatmap of lncRNA RP11-363E7.4 (F) The lncRNA RP11-363E7.4-miRNA-mRNA network extracted from global triple network. The columns represented 27 samples and the rows represented IncRNA and its neighbor mRNAs. GCLMN: gastric cancer-related lncRNAmRNA network.

Fig. 9. Analyses of GC-associated lncRNAs from network and RWR analyses. (A) The triple network of lncRNA RP11-363E7.4 extracted from global triple network. (B) The triple network of lncRNA RP11613D13.8 extracted from global triple network. GC: gastric cancer; RWR: random walk with restart analyses.

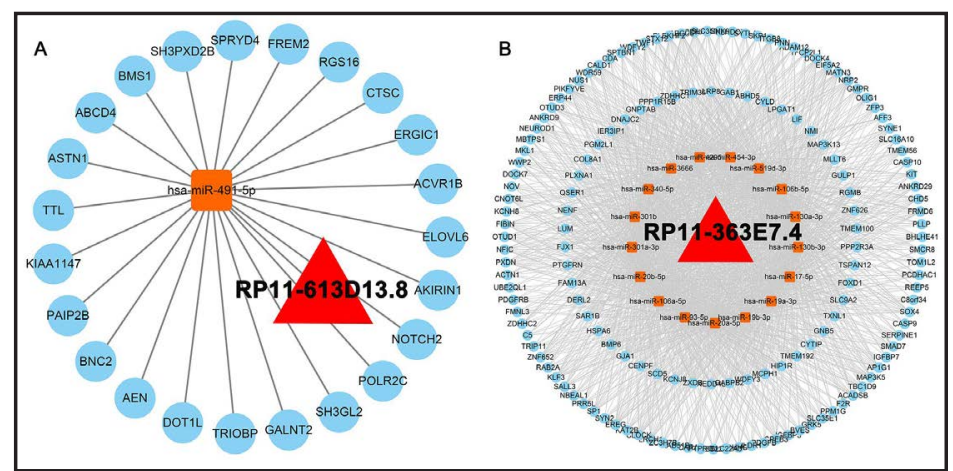


Fig. 10. LncRNA RP11-363E7.4 was downregulated in GC samples. (A) The expression level of lncRNA RP11-363E7.4 was evaluated by qPCR in 5 GC samples against corresponding adjacent noncancerous samples. Data are presented as $2^{-\Delta \Delta \mathrm{CT}}$. (B) The reannotation expression level of IncRNA RP11-363E7.4 was

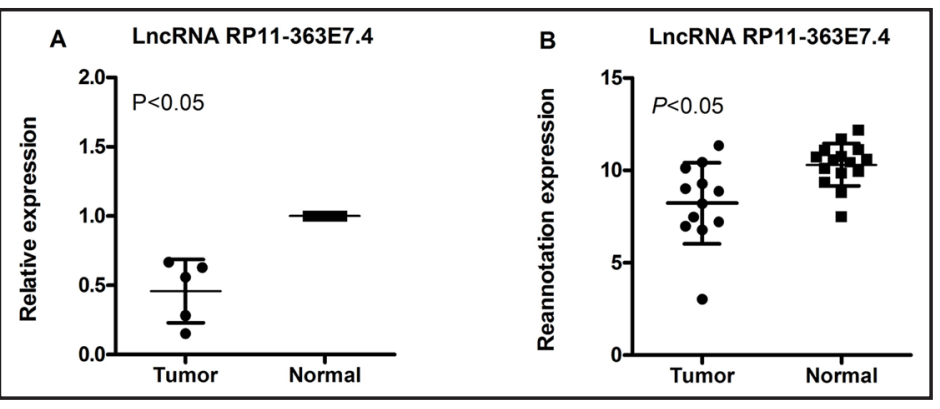
downregulated in $12 \mathrm{GC}$ tissues compared with 15 normal tissues in the microarray. $p$ values were obtained by paired $t$-test $(\mathrm{p}<0.05)$.
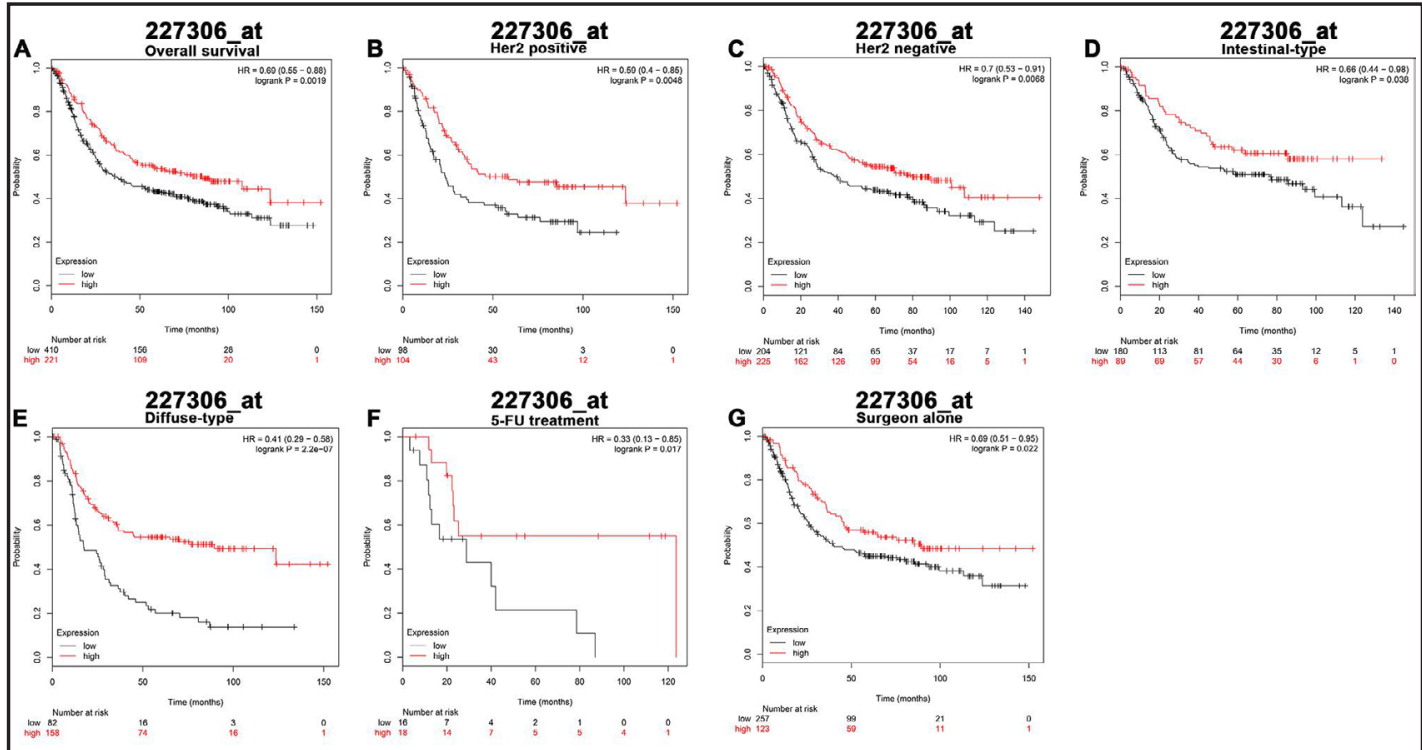

Fig. 11. Kaplan-Meier survival curves for LncRNA RP11-363E7.4. The probe 227306_at reprents IncRNA RP11-363E7.4. (A) Survival curves are plotted for all GC patients $(n=631)$. (B) Survival curves are plotted for HER2-positive patients $(n=202)$. (C) Survival curves are plotted for HER2-negative patients $(n=429)$. (D) Survival curves are plotted for intestinal type patients $(\mathrm{n}=269)$. (E) Survival curves are plotted for diffuse type patients $(n=240)$. (F) Survival curves are plotted for 5-FU treatment patients $(n=34)$. $(G)$ Survival curves are plotted for surgeon alone patients $(n=380)$. Horizontal axis: overall survival time, days; Vertical axis: survival function. HR: Hazard ratio; CI: confidence interval.

\section{Confirmation of IncRNA RP11-363E7.4 using qPCR}

To further confirm the expression levels of lncRNA RP11-363E7.4 in GC, we performed qPCR on five pairs of GC tissues and their corresponding non-cancerous tissues. The results showed that the expression level of IncRNA RP11-363E7.4 was decreased in GC tissues compared with that of the adjacent tissues. These data confirmed the microarray results, which indicated that lncRNA RP11-363E7.4 might be an important molecule in gastric carcinogenesis (Fig. 10A and 10B).

\section{The prognostic value of LncRNA RP11-363E7.4}

In our study, the probe 227306_at could represent IncRNA RP11-363E7.4. To further evaluate the prognostic value of IncRNA RP11-363E7.4, we performed online KaplanMeier analyses of GC patients. High expression of lncRNA RP11-363E7.4 was found to be correlated to better overall survival (OS) for GC patients ( $p=0.0019$ ) (Fig. 11A). Next, we investigated the correlation between IncRNA RP11-363E7.4 and other clinicopathological 

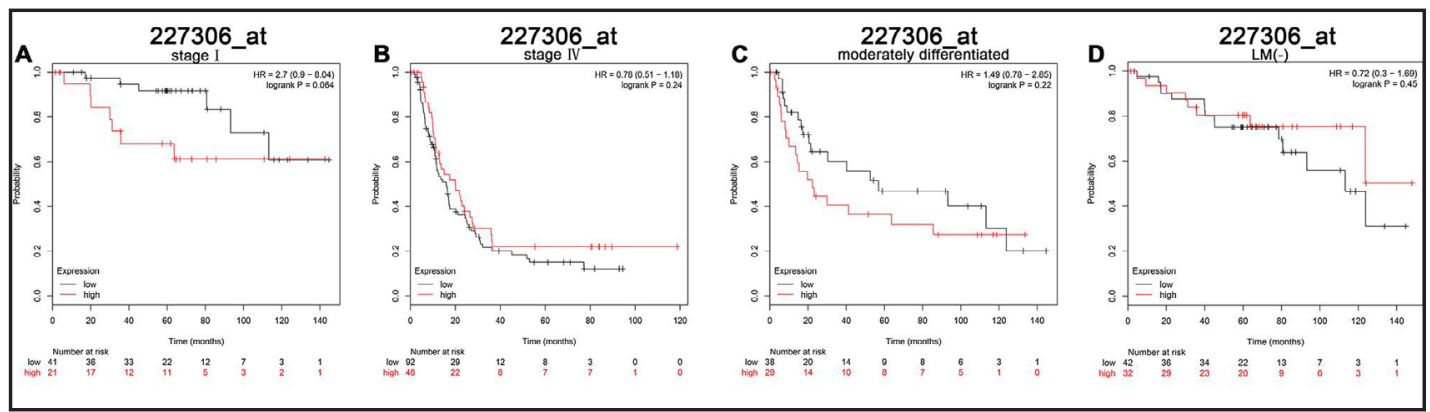

Fig. 12. Kaplan-Meier survival curves for LncRNA RP11-363E7.4. The probe 227306_at reprents lncRNA RP11-363E7.4. (A) Survival curves are plotted for stage I GC patients ( $n=62$ ). (B) Survival curves are plotted for stage IV GC patients $(n=140)$. (C) Survival curves are plotted for moderately differentiated patients $(n=67)$. (D) Survival curves are plotted for LM (-) GC patients $(n=74)$. Horizontal axis: overall survival time, days; Vertical axis: survival function. HR: Hazard ratio; CI: confidence interval; GC: gastric cancer; LM (-): lymphatic metastasis-negative.

Fig. 13. Kaplan-Meier survival curves for LncRNA RP11-363E7.4. The probe 227306_at reprents lncRNA RP11-363E7.4.

Survival curves are plotted for breast cancer patients $(n=1764)$. (B) Survival curves are plotted for lung cancer patients $(\mathrm{n}=1145)$.

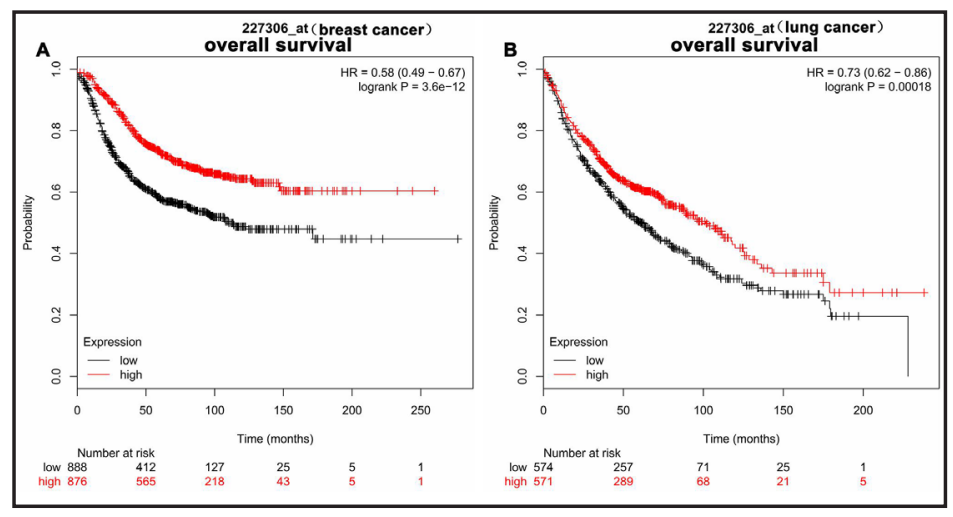

features, including clinical stages, pathological grade, lymph node metastasis status, HER2 status, Lauren classification, and different choices of treatments. Our results showed the high expression of IncRNA RP-11363E7.4 was associated with better OS regardless of the HER2 status, Lauren classification, and different treatments in GC patients (Fig. 11B-11G). The increased expression of IncRNA RP-11363E7.4 was correlated with worse OS in stage I and stage IV, moderate differentiated-type, and lymphatic metastasis-negative GC patients (Fig. 12). To testify whether IncRNA RP-11363E7.4 could be a useful biomarker for predicting OS in other cancers, we performed Kaplan-Meier analyses in breast cancer patients and lung cancer patients. The result showed it was decreased in these two cancers, and its high expression was correlated with better OS as well (Fig. 13). These findings provide powerful evidence to support our qPCR results.

\section{Discussion}

Currently, ceRNA has become an accepted mechanism for IncRNAs functions [23]. The current hypothesis is that many types of RNA transcripts interact with each other through competitive binding to miRNA response elements [23]. However, few studies have reported the IncRNA-miRNA-mRNA regulatory mechanism in GC.

In this study, we investigated the molecular mechanism of GC by constructing lncRNAmiRNA-mRNA regulatory networks based on the ceRNA hypothesis. To accomplish this, a global triple network was constructed by combining differentially expressed lncRNAs and mRNAs. In the next step, we mapped the GC related lncRNAs into a triple network to 
construct a new subnetwork of GCLMN. This new regulatory network contained six IncRNAs, 332 mRNAs, and 3, 707 edges. To further explore the key regulators in this subnetwork, we evaluated the topological features and finally identified two important lncRNAs, RP11363E7.4 and RP11-834C11.4. When we performed the RWR to the GCLMN, two key regulators were also identified, lncRNA RP11-363E7.4 and lncRNA RP613D13.8. Surprisingly, we found that IncRNA PR11-363E7.4 appeared two times using different methods, which indicated lncRNA PR11-363E7.4 might be more important than the other lncRNAs in the pathogenesis of GC.

To further investigate these three lncRNAs (RP11-363E7.4, RP11-834C11.4, and RP613D13.8), we searched the literature and found one report on IncRNA RP11-363E7.4. Wang et al. reported that the expression level of IncRNA RP11-363E7.4 was elevated in cisplatin-treated HepG2 cells as assessed by microarray analyses, and finally confirmed with qPCR. Further experiments showed that IncRNA RP11-363E7.4 positively regulated CDKN1A, TP53I3 and PPM1D at the gene level [52]. In our study, we confirmed with qPCR that lncRNA RP-11363E7.4 had decreased expression in five GC tissues. Next, we analyzed the lncRNA RP-11363E7.4-related gene function and the corresponding pathway with GO and KEGG enrichment analyses. The results using GO biological process terms showed some important signal transduction pathways for cancers, which indicated these genes might be critical in tumorigenesis. The KEGG pathway analyses showed that lncRNA RP11-363E7.4 was mainly involved in the PI3K signaling pathway and in the regulation of the actin cytoskeleton. Both pathways have been validated by numerous studies of GC samples, indicating lncRNA RP11363E7.4 may affect GC through these two pathways. When the GCLMN was extracted from the global triple network, we further analyzed this subnetwork and found three miRNAs (miR106a-5p, miR-17-5p, and miR-19b-3p) were closely associated with lncRNA RP11-363E7.4. Analyses of the literature revealed that these miRNAs (miR-17-5p, miR-19b-3p, and miR$106 \mathrm{a}-5 \mathrm{p}$ ) have been reported in GC, and have been shown to exhibit abnormal expression in GC tissues and have played roles in the development of GC. A study conducted by Qu et al. showed that miR-17-5p was highly expressed in six pairs of gastric tissues, and further experiments demonstrated that miR-17-5p promoted tumor proliferation and migration by negatively regulating TGFBR2. Zhang et al. showed that the expression of circulating miR-19b-3p in the plasma was beneficial for distinguishing normal from GC patients with different TNM stages and differentiation grades. Xia et al. reported that miR-106a-5p was highly expressed in GC tissues, and a more recent study demonstrated that the knockdown of miR-106a-5p in GC lines could effectively increase the expression of FER1L4 and PTEN at the mRNA levels. We discovered that additional protein coding genes in the miRNA-lncRNAmRNA regulators have been validated, which play key roles in GC, such as EREG, IGFBP7, NPR2, PDGFB, CLOCK, EIF5A2, and KAT2B, which are important in the regulation of the miR17-5p/miR-106a-5p-RP11-363E7.4-gene. Based on the foregoing results, we speculated there may be a regulatory network that participates in the pathogenesis of GC to include lncRNA RP11-363E7.4-miR-106a-5p-mRNA, IncRNA RP11-363E7.4-miR-17-5p-mRNA, and lncRNA RP11-363E7.4-miR-19b-3p-mRNA. However, the specific mechanism of these new regulatory networks must be validated in vitro and in vivo.

We found no reported studies on IncRNA RP11-834C11.4, therefore, the expression and mechanism of IncRNA RP11-834C11.4 in GC remain unclear. Based on the ceRNA hypothesis, we therefore further investigated the IncRNA RP11-834C11.4 neighbor mRNAs. By searching for the miRNAs related to lncRNA RP11-834C11.4, we found miR133b was not only related to GC, but was also associated with lncRNA RP11-834C11.4. A study by Yang et al. showed that the expression levels of miR-133b were downregulated in GC tissues and cells, and this significantly inhibited the proliferation, metastasis, and invasion of GC cells by targeting FBN1 [29]. Thus, we speculated that IncRNA RP11-834C11.4 participated in GC through competitive interactions with miR133b, leading to low expression of miR-133b, followed by the upregulation of FBN1. Further investigations of the regulatory relationships among lncRNA RP11-834C11.4, miR133b, and FBN1 are necessary. 


\section{Cellular Physiology Cell Physiol Biochem 2018;47:1025-1041 \begin{tabular}{l|l} 
DOI: 10.1159/000490168 & and Biochemistry \\
Published online: May 30, 2018 & $\begin{array}{l}\text { O 2018 The Author(s). Published by S. Karger AG, Basel } \\
\text { www.karger.com/cpb }\end{array}$
\end{tabular} \\ Wang et al.: Network Analyses Identifies IncRNA RP11-363E7.4 as an Important Gastric Cancer Regulator}

Similar to the IncRNA RP11-834C11.4, there are also no reports regarding lncRNA RP11613D13.8, therefore its functions in GC remain unknown. In Fig. 11B, we show the close relationship between IncRNA RP11-613D13.8 and miR491-5p. Previous studies regarding miR-491-5p have demonstrated that it played an important role in different cancers, including GC [49, 53-56], and could also have critical roles in a variety of cellular processes, including cell proliferation, tumor metastasis, tumor invasion, cell apoptosis, and the cell cycle. Sun et al. reported that the expression of miR-491-5p was markedly downregulated in GC, which inhibited cell proliferation by disrupting the cell cycle and promoting apoptosis in GC cells via targeting Wnt3a [49]. Thus, we speculate that lncRNA RP11-613D13.8 regulates its corresponding miRNA (miR491-5p) and targeted mRNAs through the ceRNA mechanism as hypothesized previously. Further investigation both in vitro and in vivo are required to determine the exact mechanisms of IncRNA RP11-613D13.8.

Recently, Xia and colleagues (2014) performed IncRNA microarray analyses and mRNA expression profiles with six samples of GC, and constructed a miRNA-lncRNA-mRNA triple interaction network, which helped identify eight lncRNAs (AC009499.1, GACAT1, GACAT3, H19, LINC00152, AP000288.2, FER1L4, and RP4-620F22.3) and nine miRNAs (miR-18a-5p, miR-18b-5p, miR-19a-3p, miR-20b-5p, miR-106a-5p, miR-106b-5p, miR-31-5p, miR-139-5p, and miR-195-5p) significantly associated with multiple cancer types, including GC. Although our work did not share the same risk as the lncRNAs induced by the heterogeneity of the IncRNA reference data, both studies determined that miR-19a-3p, miR-20b-5p, miR-106a$5 p$, and miR-106b-5p were significantly associated with GC by different annotated processes. The straight annotation method used by Xia and colleagues and the re-annotation method used in this study can be considered complementary research strategies in the identification of lncRNAs linked to GC risk and the potential regulatory mechanism.

Although we have provided an effective approach for discovering crucial GC-related lncRNAs and identifying a novel regulatory mechanism through the network, some limitations exist in our study. The present study was a reannotation, and we could not cover all IncRNAs. There were 3, 400 lncRNAs obtained after reannotation, but many "hot" IncRNAs were not screened in this network, including MALTA1 [57, 58], HOTAIR [59], MEG3 [60], and H19 [8, 61]. Our future studies will focus on amplifying the samples and concentrate on collecting the experimental evidence to validate our ceRNA hypothesis. Finally, our results could be improved by including more complete expression data for IncRNAs and mRNAs in GC.

Taken together, our study has successfully identified crucial lncRNAs in GC by bioinformatics analyses. The regulatory network we have provided may suggest a new approach to IncRNA studies in GC, and may identify useful therapeutic targets for GC in the future.

\section{Acknowledgements}

This work was supported in part by the Natural Science Foundation of Heilongjiang Province of China (Grant number: H201405).

\section{Disclosure Statement}

The authors have declared that they have no conflict of interests to disclose.

\section{References}

\footnotetext{
1 Ferlay J, Soerjomataram I, Dikshit R, Eser S, Mathers C, Rebelo M, Parkin DM, Forman D, Bray F: Cancer incidence and mortality worldwide: sources, methods and major patterns in GLOBOCAN 2012 Int J Cancer 2015;136:E359-386.
} 


\section{Cellular Physiology Cell Physiol Biochem 2018;47:1025-1041

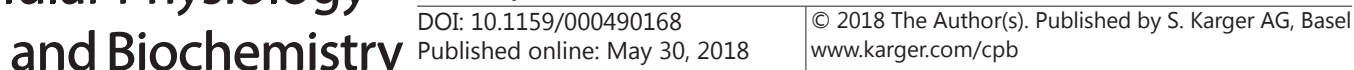

Wang et al.: Network Analyses Identifies IncRNA RP11-363E7.4 as an Important Gastric Cancer Regulator

-2 Lin XC, Zhu Y, Chen WB, Lin LW, Chen DH, Huang JR, Pan K, Lin Y, Wu BT, Dai Y, Tu ZG: Integrated analysis of long non-coding RNAs and mRNA expression profiles reveals the potential role of lncRNAs in gastric cancer pathogenesis. Int J Oncol 2014;45:619-628.

-3 Ji N, Yu JW, Ni XC, Wu JG, Wang SL, Jiang BJ: Bone marrow-derived mesenchymal stem cells increase drug resistance in CD133-expressing gastric cancer cells by regulating the PI3K/AKT pathway. Tumour Biol 2016;37:14637-14651.

-4 Katoh H, Ishikawa S: Genomic pathobiology of gastric carcinoma. Pathol Int 2017;67:63-71.

5 Du C, Li DQ, Li N, Chen L, Li SS, Yang Y, Hou MX, Xie MJ, Zheng ZD: DDX5 promotes gastric cancer cell proliferation in vitro and in vivo through mTOR signaling pathway. Sci Rep 2017;7:42876.

6 Song G, Shen Y, Ruan Z, Li X, Chen Y, Yuan W, Ding X, Zhu L, Qian L: LncRNA-uc.167 influences cell proliferation, apoptosis and differentiation of P19 cells by regulating Mef2c. Gene 2016;590:97-108.

7 Zhao J, Liu Y, Zhang W, Zhou Z, Wu J, Cui P, Zhang Y, Huang G: Long non-coding RNA Linc00152 is involved in cell cycle arrest, apoptosis, epithelial to mesenchymal transition, cell migration and invasion in gastric cancer. Cell Cycle 2015;14:3112-3123.

-8 Yan J, Zhang Y, She Q, Li X, Peng L, Wang X, Liu S, Shen X, Zhang W, Dong Y, Lu J, Zhang G: Long Noncoding RNA H19/miR-675 Axis Promotes Gastric Cancer via FADD/Caspase 8/Caspase 3 Signaling Pathway. Cell Physiol Biochem 2017;42:2364-2376.

-9 Yan K, Tian J, Shi W, Xia H, Zhu Y: LncRNA SNHG6 is Associated with Poor Prognosis of Gastric Cancer and Promotes Cell Proliferation and EMT through Epigenetically Silencing p27 and Sponging miR-101-3p. Cell Physiol Biochem 2017;42:999-1012.

10 Hammerle M, Gutschner T, Uckelmann H, Ozgur S, Fiskin E, Gross M, Skawran B, Geffers R, Longerich T, Breuhahn K, Schirmacher P, Stoecklin G, Diederichs S: Posttranscriptional destabilization of the liver-specific long noncoding RNA HULC by the IGF2 mRNA-binding protein 1 (IGF2BP1). Hepatology 2013;58:17031712.

-11 Yoon JH, Abdelmohsen K, Gorospe M: Posttranscriptional gene regulation by long noncoding RNA. J Mol Biol 2013;425:3723-3730.

-12 Ma C, Wu G, Zhu Q Liu H, Yao Y, Yuan D, Liu Y, Lv T, Song Y: Long intergenic noncoding RNA 00673 promotes non-small-cell lung cancer metastasis by binding with EZH2 and causing epigenetic silencing of HOXA5 Oncotarget 2017;8:32696-32705.

13 Hu Y, Sun X, Mao C, Guo G, Ye S, Xu J, Zou R, Chen J, Wang L, Duan P, Xue X: Upregulation of long noncoding RNA TUG1 promotes cervical cancer cell proliferation and migration. Cancer Med 2017;6:471-482.

14 Li D, Li H, Yang Y, Kang L: Long Noncoding RNA Urothelial Carcinoma Associated 1 Promotes the Proliferation and Metastasis of Human Lung Tumor Cells by Regulating MicroRNA-144 Oncol Res 2017;10.3727/09650 $4017 X 15009792179602$

15 Xu S, Kong D, Chen Q Ping Y, Pang D: Oncogenic long noncoding RNA landscape in breast cancer. Mol Cancer 2017;16:129.

16 Xu Y, Qiu M, Chen Y, Wang J, Xia W, Mao Q, Yang L, Li M, Jiang F, Xu L, Yin R: Long noncoding RNA, tissue differentiation-inducing nonprotein coding RNA is upregulated and promotes development of esophageal squamous cell carcinoma. Dis Esophagus 2016;29:950-958.

$\checkmark 17$ Lu S, Zhou J, Sun Y, Li N, Miao M, Jiao B, Chen H: The noncoding RNA HOXD-AS1 is a critical regulator of the metastasis and apoptosis phenotype in human hepatocellular carcinoma. Mol Cancer 2017;16:125.

18 Lian Y, Xu Y, Xiao C, Xia R, Gong H, Yang P, Chen T, Wu D, Cai Z, Zhang J, Wang K: The pseudogene derived from long non-coding RNA DUXAP10 promotes colorectal cancer cell growth through epigenetically silencing of p21 and PTEN. Sci Rep 2017;7:7312.

19 Yu B, Ye X, Du Q, Zhu B, Zhai Q Li XX: The Long Non-Coding RNA CRNDE Promotes Colorectal Carcinoma Progression by Competitively Binding miR-217 with TCF7L2 and Enhancing the Wnt/beta-Catenin Signaling Pathway. Cell Physiol Biochem 2017;41:2489-2502.

20 Chen Z, Liu H, Yang H, Gao Y, Zhang G, Hu J: The long noncoding RNA, TINCR, functions as a competing endogenous RNA to regulate PDK1 expression by sponging miR-375 in gastric cancer. Onco Targets Ther 2017;10:3353-3362.

21 Lei K, Liang X, Gao Y, Xu B, Xu Y, Li Y, Tao Y, Shi W, Liu J: Lnc-ATB contributes to gastric cancer growth through a MiR-141-3p/TGFbeta2 feedback loop. Biochem Biophys Res Commun 2017;484:514-521.

22 Xia T, Chen S, Jiang Z, Shao Y, Jiang X, Li P, Xiao B, Guo J: Long noncoding RNA FER1L4 suppresses cancer cell growth by acting as a competing endogenous RNA and regulating PTEN expression. Sci Rep 2015;5:13445. 


\section{Cellular Physiology Cell Physiol Biochem 2018;47:1025-1041 \begin{tabular}{ll|l} 
DOI: 10.1159/000490168 & O 2018 The Author(s). Published by S. Karger AG, Basel \\
www.karger.com/cpb
\end{tabular} and Biochemistry}

Wang et al.: Network Analyses Identifies IncRNA RP11-363E7.4 as an Important Gastric Cancer Regulator

23 Salmena L, Poliseno L, Tay Y, Kats L, Pandolfi PP: A ceRNA hypothesis: the Rosetta Stone of a hidden RNA language? Cell 2011;146:353-358.

-24 Zhao L, Guo H, Zhou B, Feng J, Li Y, Han T, Liu L, Li L, Zhang S, Liu Y, Shi J, Zheng D: Long non-coding RNA SNHG5 suppresses gastric cancer progression by trapping MTA2 in the cytosol. Oncogene 2016;35:57705780 .

25 Zhao L, Han T, Li Y, Sun J, Zhang S, Liu Y, Shan B, Zheng D, Shi J: The lncRNA SNHG5/miR-32 axis regulates gastric cancer cell proliferation and migration by targeting KLF4. FASEB J 2017;31:893-903.

-26 Zhang R, Guo Y, Ma Z, Ma G, Xue Q, Li F, Liu L: Long non-coding RNA PTENP1 functions as a ceRNA to modulate PTEN level by decoying miR-106b and miR-93 in gastric cancer. Oncotarget 2017;8:26079-26089.

-27 Wang Q, Wen YG, Li DP, Xia J, Zhou CZ, Yan DW, Tang HM, Peng ZH: Upregulated INHBA expression is associated with poor survival in gastric cancer. Med Oncol 2012;29:77-83.

28 Song C, Zhang J, Liu Y, Pan H, Qi HP, Cao YG, Zhao JM, Li S, Guo J, Sun HL, Li CQ: Construction and analysis of cardiac hypertrophy-associated IncRNA-mRNA network based on competitive endogenous RNA reveal functional lncRNAs in cardiac hypertrophy. Oncotarget 2016;7:10827-10840.

29 Tusher VG, Tibshirani R, Chu G: Significance analysis of microarrays applied to the ionizing radiation response. Proc Natl Acad Sci U S A 2001;98:5116-5121.

-30 Yang JH, LiJH, Shao P, Zhou H, Chen YQ, Qu LH: starBase: a database for exploring microRNA-mRNA interaction maps from Argonaute CLIP-Seq and Degradome-Seq data. Nucleic Acids Res 2011;39:D202-209.

-31 Kohler S, Bauer S, Horn D, Robinson PN: Walking the interactome for prioritization of candidate disease genes. Am J Hum Genet 2008;82:949-958.

-32 Le DH, Kwon YK: Neighbor-favoring weight reinforcement to improve random walk-based disease gene prioritization. Comput Biol Chem 2013;44:1-8.

-33 Huang M, Hou J, Wang Y, Xie M, Wei C, Nie F, Wang Z, Sun M: Long Noncoding RNA LINC00673 Is Activated by SP1 and Exerts Oncogenic Properties by Interacting with LSD1 and EZH2 in Gastric Cancer. Mol Ther 2017;25:1014-1026.

-34 Vale Rodrigues R, Santos F, Pereira da Silva J, Francisco I, Claro I, Albuquerque C, Lemos MM, Limbert M, Dias Pereira A: A case of multiple gastrointestinal stromal tumors caused by a germline KIT gene mutation (p.Leu576Pro). Fam Cancer 2017;16:267-270.

-35 Cheng TY, Wu MS, Hua KT, Kuo ML, Lin MT: Cyr61/CTGF/Nov family proteins in gastric carcinogenesis. World J Gastroenterol 2014;20:1694-1700.

-36 Hu ML, Yeh KT, Lin PM, Hsu CM, Hsiao HH, Liu YC, Lin HY, Lin SF, Yang MY: Deregulated expression of circadian clock genes in gastric cancer. BMC Gastroenterol 2014;14:67.

37 Huang da W, Sherman BT, Lempicki RA: Systematic and integrative analysis of large gene lists using DAVID bioinformatics resources. Nat Protoc 2009;4:44-57.

-38 Livak KJ, Schmittgen TD: Analysis of relative gene expression data using real-time quantitative PCR and the 2(-Delta Delta C(T)) Method. Methods 2001;25:402-408.

-39 Szasz AM, Lanczky A, Nagy A, Forster S, Hark K, Green JE, Boussioutas A, Busuttil R, Szabo A, Gyorffy B: Crossvalidation of survival associated biomarkers in gastric cancer using transcriptomic data of 1, 065 patients. Oncotarget 2016;7:49322-49333.

40 Cui Y, Wang Y, Li H, Li Q, Yu Y, Xu X, Xu B, Liu T: Asparaginyl endopeptidase promotes the invasion and metastasis of gastric cancer through modulating epithelial-to-mesenchymal transition and analysis of their phosphorylation signaling pathways. Oncotarget 2016;7:34356-34370.

41 Lan T, Pang J, Wu Y, Zhu M, Yao X, Wu M, Qian H, Zhang Z, Gao J, Chen Y: Cross-linked hyaluronic acid gel inhibits metastasis and growth of gastric and hepatic cancer cells: in vitro and in vivo studies. Oncotarget 2016;7:65418-65428.

42 Fujimoto D, Ueda Y, Hirono Y, Goi T, Yamaguchi A: PAR1 participates in the ability of multidrug resistance and tumorigenesis by controlling Hippo-YAP pathway. Oncotarget 2015;6:34788-34799.

43 Pang L, Li JF, Su L, Zang M, Fan Z, Yu B, Wu X, Li C, Yan M, Zhu ZG, Liu B: ALEX1, a novel tumor suppressor gene, inhibits gastric cancer metastasis via the PAR-1/Rho GTPase signaling pathway. J Gastroenterol 2017;10.1007/s00535-017-1329-y

44 Zhu X, Jiang X, Li A, Sun Y, Liu Y, Sun X, Feng X, Li S, Zhao Z: S-allylmercaptocysteine suppresses the growth of human gastric cancer xenografts through induction of apoptosis and regulation of MAPK and PI3K/Akt signaling pathways. Biochem Biophys Res Commun 2017;10.1016/j.bbrc.2017.06.107 


\section{Cellular Physiology Cell Physiol Biochem 2018;47:1025-1041 \begin{tabular}{l|l} 
DOI: 10.1159/000490168 & O 2018 The Author(s). Published by S. Karger AG, Basel \\
www.karger.com/cpb
\end{tabular}}

Wang et al.: Network Analyses Identifies IncRNA RP11-363E7.4 as an Important Gastric Cancer Regulator

45 Liu Y, Zhang X, Zhang Y, Hu Z, Yang D, Wang C, Guo M, Cai Q: Identification of miRNomes in human stomach and gastric carcinoma reveals miR-133b/a-3p as therapeutic target for gastric cancer. Cancer Lett 2015;369:5866.

46 Shen B, Yu S, Zhang Y, Yuan Y, Li X, Zhong J, Feng J: miR-590-5p regulates gastric cancer cell growth and chemosensitivity through RECK and the AKT/ERK pathway. Onco Targets Ther 2016;9:6009-6019.

$\$ 47$ Ohshima K, Inoue K, Fujiwara A, Hatakeyama K, Kanto K, Watanabe Y, Muramatsu K, Fukuda Y, Ogura S, Yamaguchi K, Mochizuki T: Let-7 microRNA family is selectively secreted into the extracellular environment via exosomes in a metastatic gastric cancer cell line. PLoS One 2010;5:e13247.

-48 Zhang Z, Liu S, Shi R, Zhao G: miR-27 promotes human gastric cancer cell metastasis by inducing epithelialto-mesenchymal transition. Cancer Genet 2011;204:486-491.

49 Sun R, Liu Z, Tong D, Yang Y, Guo B, Wang X, Zhao L, Huang C: miR-491-5p, mediated by Foxi1, functions as a tumor suppressor by targeting Wnt3a/beta-catenin signaling in the development of gastric cancer. Cell Death Dis 2017;8:e2714.

50 Zhang J, Song Y, Zhang C, Zhi X, Fu H, Ma Y, Chen Y, Pan F, Wang K, Ni J, Jin W, He X, Su H, Cui D: Circulating MiR-16-5p and MiR-19b-3p as Two Novel Potential Biomarkers to Indicate Progression of Gastric Cancer. Theranostics 2015;5:733-745.

51 Qu Y, Zhang H, Duan J, Liu R, Deng T, Bai M, Huang D, Li H, Ning T, Zhang L, Wang X, Ge S, Zhou L, Zhong B, Ying G, Ba Y: MiR-17-5p regulates cell proliferation and migration by targeting transforming growth factor-beta receptor 2 in gastric cancer. Oncotarget 2016;7:33286-33296.

52 Wang P, Cui J, Wen J, Guo Y, Zhang L, Chen X: Cisplatin induces HepG2 cell cycle arrest through targeting specific long noncoding RNAs and the p53 signaling pathway. Oncol Lett 2016;12:4605-4612.

53 Hanisch C, Sharbati J, Kutz-Lohroff B, Huber O, Einspanier R, Sharbati S: TFF3-dependent resistance of human colorectal adenocarcinoma cells HT-29/B6 to apoptosis is mediated by miR-491-5p regulation of IncRNA PRINS. Cell Death Discov 2017;3:16106.

54 Yin Z, Ding H, He E, Chen J, Li M: Up-regulation of microRNA-491-5p suppresses cell proliferation and promotes apoptosis by targeting FOXP4 in human osteosarcoma. Cell Prolif 2017;50:

55 Zhang Q, Li Q Xu T, Jiang H, Xu LG: miR-491-5p suppresses cell growth and invasion by targeting Notch3 in nasopharyngeal carcinoma. Oncol Rep 2016;35:3541-3547.

56 Huang WC, Chan SH, Jang TH, Chang JW, Ko YC, Yen TC, Chiang SL, Chiang WF, Shieh TY, Liao CT, Juang JL, Wang HC, Cheng AJ, Lu YC, Wang LH: miRNA-491-5p and GIT1 serve as modulators and biomarkers for oral squamous cell carcinoma invasion and metastasis. Cancer Res 2014;74:751-764.

57 Zhang Y, Chen Z, Li MJ, Guo HY, Jing NC: Long non-coding RNA metastasis-associated lung adenocarcinoma transcript 1 regulates the expression of Gli2 by miR-202 to strengthen gastric cancer progression. Biomed Pharmacother 2017;85:264-271.

58 Li L, Geng Y, Feng R, Zhu Q, Miao B, Cao J, Fei S: The Human RNA Surveillance Factor UPF1 Modulates Gastric Cancer Progression by Targeting Long Non-Coding RNA MALAT1. Cell Physiol Biochem 2017;42:2194-2206.

59 Pan W, Liu L, Wei J, Ge Y, Zhang J, Chen H, Zhou L, Yuan Q Zhou C, Yang M: A functional lncRNA HOTAIR genetic variant contributes to gastric cancer susceptibility. Mol Carcinog 2016;55:90-96.

60 Peng W, Si S, Zhang Q, Li C, Zhao F, Wang F, Yu J, Ma R: Long non-coding RNA MEG3 functions as a competing endogenous RNA to regulate gastric cancer progression. J Exp Clin Cancer Res 2015;34:79.

61 Liu G, Xiang T, Wu QF, Wang WX: Long Noncoding RNA H19-Derived miR-675 Enhances Proliferation and Invasion via RUNX1 in Gastric Cancer Cells. Oncol Res 2016;23:99-107. 